\title{
INDOLEAMINE 2,3 DIOXYGENASE AS AN IMMUNOTHERAPEUTIC TARGET BRINGS A NEW HOPE FOR CANCER PATIENTS
}

\author{
Kashif Asghar ${ }^{1}$, Asif Loya ${ }^{2}$ \\ ${ }^{1}$ Department of Basic Sciences Research, Shaukat Khanum Memorial Cancer Hospital and Research Centre, \\ Lahore, Pakistan, ${ }^{2}$ Department of Pathology, Shaukat Khanum Memorial Cancer Hospital and Research Centre, \\ Lahore, Pakistan
}

Received: 22 May 2018 / Accepted: 17 September 2018

\begin{abstract}
Therapeutic manipulation of immune system in cancer has been an extensive area of research in the field of oncoimmunology. Immunotherapy helps the immune system to combat against cancer. Tumour cells take an edge of immunosuppressive mechanisms and inhibit antitumour immune responses. Indoleamine 2,3-dioxygenase (IDO) is an immunosuppressive enzyme which is involved in tumour immune escape mechanism in various cancers. IDO can degrade the tryptophan into kynurenines and has an ability to enhance the immune tolerance through mammalian target of rapamycin pathway general control nonderepressible 2 (GCN2) pathway and induction of regulatory T (T-regs) cells. IDO-induced T-regs suppress the local immune responses in the tumour microenvironment and promote metastasis. IDO overexpression in various cancers is associated with poor prognosis. Several preclinical and clinical trials have been proceeding and recommend that IDO inhibitor may be an influential tool against a wide range of cancers. IDO inhibitors as adjuvant therapeutic agents may also have clinical implications. Thus, IDO has the potential to be used as an immunotherapeutic target. This review discusses the promising role of IDO in cancer and its implication in immunotherapy.
\end{abstract}

Key words: Breast cancer, colorectal cancer, haematological malignancies, immunotherapy, indoleamine 2,3-dioxygenase, pancreatic cancer, prostate cancer

\section{Introduction}

Immunotherapy uses specific parts of an individual's immune system to fight against cancer. Cancer immunotherapy is promptly progressing and now is considered as the "fifth pillar" of cancer therapy. ${ }^{[1]}$ Immunotherapeutic approaches include adoptive cellular immunotherapy, cancer vaccines, oncolytic viruses and immune checkpoint blockade. ${ }^{[2]}$ It has been established that the defective immune system plays a critical role in cancer development. ${ }^{[3]}$ Some malignant cells have the capacity to manipulate their own characteristics as well as cells in their microenvironment to form "successful" tumours thus evading the tumour immunosurveillance system. ${ }^{[1]}$ Cancer cells can escape the immune attack through various complex mechanisms, including

Correspondence: Dr. Kashif Asghar, Department of Basic Sciences Research, Shaukat Khanum Memorial Cancer Hospital and Research Centre, Lahore, Pakistan.

Email: kashifasghar@skm.org.pk tumour induced immunosuppression through the upregulation of immunosuppressive enzymes, such as indoleamine 2,3-dioxygenase (IDO/IDO1). ${ }^{[4,5]}$ IDO is a heme-containing enzyme involved in tryptophan catabolism. ${ }^{[6]}$ IDO induces immunosuppression through tryptophan degradation and generation of tryptophan metabolites. ${ }^{[6]}$ Tryptophan degradation by IDO directly affects T-cell proliferation through the activation of the GCN2 kinase pathway. ${ }^{[7]}$ Tryptophan metabolites (Kynurenines) also have the potential to induce apoptosis in lymphocytes. ${ }^{[8]}$ T-cell immunity may be inhibited by IDO through induction of differentiation and maturation of T-regs. ${ }^{[9]}$ Overexpression of IDO is involved in immunosuppression and tolerance. ${ }^{[10]}$ IDO producing cells are found at various immune tolerance sites, including thymus, placenta, anterior chamber of eye, mucosa of gut and epididymis..$^{[11-13]}$

IDO is expressed by human monocyte-derived macrophages and dendritic cells (DC). ${ }^{[14,15]}$ Role of IDO 
and its mode of action on cancer growth and immune evasion are still nascent in human cancer research. Numerous studies have documented a probable association between various clinical or immunologic parameters and IDO expression. Enhanced IDO expression in human cancer patients is linked with metastasis and predicts poor patient survival. ${ }^{[16]}$ IDO is involved in tumour immune escape. ${ }^{[17]}$ This review describes the involvement of IDO in various malignancies and proposes future prospects of IDO as immunotherapeutic target.

\section{IDO in Breast Cancer}

IDO protein is expressed in solid tumours such as breast cancer. ${ }^{[18]}$ The involvement of IDO in breast cancer pathogenesis has been outlined in Table 1. IDO overexpression has an autonomous prognostic significance in basal-like breast carcinoma (BLBC) and is linked with morphological medullary features. Medullary breast carcinoma $(\mathrm{MBC})$ has a better prognosis than non-MBC, but IDO is overexpressed at mRNA level in BLBC and $\mathrm{MBC}$ as compared to non-MBC. Tumour-infiltrating lymphocytes (TILs) reported in both MBC and BLBC. IDO expression is correlated with TILs. ${ }^{[19]}$ IDO is involved in metastasis formation and tumour immune escape in murine breast cancer cell lines as well. $\mathrm{IDO}^{+}$tumour grows faster than $\mathrm{IDO}^{-}$tumours in immunodeficient severe combined immunodeficiency mice. IDO1 is involved in breast tumour growth and spontaneous pulmonary metastasis formation. ${ }^{[20]}$ Soliman et al. analysed an expression of IDO protein in 203 breast cancer cases. IDO overexpression was observed in $\mathrm{ER}^{+}$(oestrogen receptor) tumour as compared to the $\mathrm{ER}^{-}$tumours $(P=0.0064)$. This study gave a new dimension to the ongoing clinical trials of IDO inhibitors in the metastatic breast cancer. They proposed further studies to understand the complicated role of IDO in breast cancer progression at different stages of the disease. ${ }^{[21]}$

Another murine breast cancer model research provided evidence that gene silencing of IDO is a potent approach to enhance the efficacy of DC-based cancer immunotherapy. IDO-silenced DCs improve cytotoxic T lymphocyte activity and tumour antigen-specific T-cell proliferation. IDO targeted inhibition in DCs may be a convenient resolution to boost the efficiency of DC vaccine in clinics. ${ }^{[22]}$

IDO overexpression was found in myeloid-derived suppressive cells (MDSCs), extracted from fresh breast

Table 1: Comprehensive review of indoleamine 2,3-dioxygenase involvement in breast cancer

\begin{tabular}{|c|c|c|}
\hline Year & Investigator & Description \\
\hline 2012 & Jacquemier et al. ${ }^{[19]}$ & $\begin{array}{l}\text { IDO overexpression is associated with morphological medullary features and has indepen- } \\
\text { dent prognostic value in BLBC }\end{array}$ \\
\hline 2012 & Levina et al. ${ }^{[20]}$ & $\begin{array}{l}\text { IDO1 expression has immunological and non-immunological effects on breast tumour } \\
\text { growth and spontaneous pulmonary metastasis formation }\end{array}$ \\
\hline 2013 & Soliman et al. ${ }^{[21]}$ & Higher IDO expression is observed in tumours with ER+phenotype than ER - tumours \\
\hline 2013 & Zheng et al. ${ }^{[22]}$ & IDO gene silencing may enhance the efficacy of (DC)-based cancer immunotherapeutic \\
\hline 2013 & Yu et al. ${ }^{[23]}$ & MDSCs suppress the anti-tumour immune responses by IDO expression in breast cancer \\
\hline 2014 & Isla Larrain et al. ${ }^{[24]}$ & $\begin{array}{l}\text { In vitro and in silico gene expression revealed that IDO is expressed in a (TNBC) subgroup. } \\
\text { IDO is involved in the tumour immune escape }\end{array}$ \\
\hline 2015 & Salvadori et al..$^{[25]}$ & $\begin{array}{l}\text { A decreased IDO expression is noted in cultured cancer cells of breast cancer patients when } \\
\text { paclitaxel is given in combination with IDO inhibitor. It may be a new therapeutic strategy } \\
\text { for breast cancer }\end{array}$ \\
\hline 2016 & Chen et al..$^{[26]}$ & $\begin{array}{l}\text { IDO inhibitors in combination with chemotherapeutic agents improve antitumor responses } \\
\text { in breast cancer mouse model }\end{array}$ \\
\hline 2017 & Kim et al. ${ }^{[27]}$ & $\begin{array}{l}\text { IDO expression is associated with BLBC. IDO inhibition may play a key role in the treatment } \\
\text { of (BL) TNBC }\end{array}$ \\
\hline 2017 & Noonepalle et al. ${ }^{[28]}$ & $\begin{array}{l}\text { IDO1 methylation regulates anti-immune responses in breast cancer subtypes. IDO methyla- } \\
\text { tion can be used as a prognostic biomarker for IDO inhibitor based immunotherapy }\end{array}$ \\
\hline
\end{tabular}

IDO: Indoleamine 2,3-dioxygenase, BL: BASAL-like, BLBC: BL breast carcinoma, DC: Dendritic cell, MDSCs: Myeloid-derived suppressive cells, TNBC: Triple negative breast cancer 
cancer tissues. It was associated with a high frequency of forkhead box P3 (Foxp3 ${ }^{+}$T-regs in lymph node metastasis and tumours. The role of signal transducer and activator of transcription 3 (STAT3) in IDO expression and IDOdependent MDSC-mediated immunosuppression on $\mathrm{T}$ cells was reported in this study. ${ }^{[23]}$ IDO expression was detected in the sub-group of triple negative breast cancer (TNBC).

Furthermore, in silico studies verified the expression of IDO as well. ${ }^{[2]}$ 1-Methyl-DL-tryptophan (D-1MT) is an IDO inhibitor which has therapeutic significance when given in combination with chemotherapeutic agents for breast cancer treatment. ${ }^{[25]}$ Paclitaxel in combination with D-1MT decreased IDO expression in cultured cells from the breast tumour microenvironment. ${ }^{[25]}$ Similar results were obtained in a breast cancer mouse model using NLG-919 (IDO inhibitor). ${ }^{[26]}$

A recently published data revealed that IDO1 expression is linked with TNBC with the BL phenotype and increased stromal lymphocytic infiltration. IDO1 immune checkpoint blockade may play a crucial role in the treatment of patients with TNBC. ${ }^{[27]}$ Anti-immune responses exhibited by breast cancer subtypes are regulated by methylation of IDO1. IDO methylation can be used as a prognostic biomarker for IDO inhibitor based immunotherapy. ${ }^{[28]}$

\section{IDO in Colorectal Cancer (CRC)}

CRC is one of the major public health problems in the world. ${ }^{[29]}$ The role of IDO in CRC has been outlined in
Table 2. IDO1 expression is an independent prognostic factor in the pT1-4N1Mx-staged CRC. ${ }^{\left[{ }^{[0]}\right.}$ Expression was associated with metachronous metastases and overall survival. IDO1 is a promising prognostic indicator in CRC identified by Ferdinande et al. ${ }^{[30]}$ A murine model study indicates that IDO1 induces tumour proliferation and growth of neoplastic epithelium in a cell-autonomous fashion through activation of $\beta$-catenin signalling and kynurenine metabolites production. These results have considerable implications for IDO1 inhibitors as immunotherapeutic agents for IDO1- expressing colitisassociated and sporadic colonic neoplasms. ${ }^{[31]}$

Daniel et al. (2015) measured the activity of IDO in patients with CRC. Quantification of IDO enzymatic activity was performed through high-performance liquid chromatography (HPLC) in the serum of 68 patients. IDO activity was observed high in patients with CRC. They suggested an association between IDO activity and CRC; further studies on IDO activity are required to establish it as a reliable serum biomarker of $\mathrm{CRC}$. $^{[32]}$

A recently published data about IDO1 expression also revealed that it is involved in the progression of CRC and is linked with impaired clinical prognosis. In this study, they analysed the expression of IDO1 and beta-catenin proteins by immunohistochemistry on the tissue samples of 192 CRC patients. IDO1-regulated molecular pathway was demonstrated to upregulate abnormal beta-catenin expression in the nucleus and cytoplasm of CRC patients having a low mutation rate of adenomatous polyposis coli,

Table 2: Indoleamine 2,3-dioxygenase involvement in colorectal cancer

\begin{tabular}{|c|c|c|}
\hline Year & Investigator & Description \\
\hline 2012 & Ferdinande et al..$^{[30]}$ & $\begin{array}{l}\text { High IDO1 expression at the tumour invasion is associated with (CRC) progression and } \\
\text { correlates with impaired clinical outcome, implying that IDO1 is an independent prognostic } \\
\text { marker for CRC }\end{array}$ \\
\hline 2013 & Thaker et al. ${ }^{[31]}$ & $\begin{array}{l}\text { IDO1 is involved in colitis-associated tumorigenesis in mice. The epithelial cell-autonomous } \\
\text { survival advantage supported by IDO1 to colon epithelial cells demonstrate its potential as a } \\
\text { therapeutic target }\end{array}$ \\
\hline 2015 & Eyraud et al..$^{[32]}$ & $\begin{array}{l}\text { IDO activity is higher in patients with CRC compared with those without CRC. This study } \\
\text { is the first step to establish that IDO enzymatic activity quantification is a reliable serum } \\
\text { marker for CRC }\end{array}$ \\
\hline 2016 & Chen et al. ${ }^{[33]}$ & $\begin{array}{l}\text { IDO1 regulated molecular pathway led to the abnormal expression of beta-catenin in the } \\
\text { nucleus and cytoplasm of CRC patients with a low mutation rate of (APC), endorsing IDO1 is } \\
\text { an appealing target for immunotherapy in CRC }\end{array}$ \\
\hline
\end{tabular}

CRC: Colorectal cancer, APC: Adenomatous polyposis coli, IDO: Indoleamine 2,3-dioxygenase 
thus suggesting IDO1 an attractive immunotherapeutic target in CRC. ${ }^{[33]}$

Engin et al. indicated that high IDO immunostaining score is a strong predictor for lymph node metastasis. ${ }^{[29]}$ IDO expression allows cancer subsets to evade immune attack in colorectal tumour cells. However, a suitable tool for the perseverance of undetected tumour cells does not exist that may be responsible for recurrent CRC. They recommended IDO immunostaining for histopathological evaluation of CRC cases. ${ }^{[29]}$

\section{IDO in Haematological Malignancies}

Haematological malignancies are cancers that affect the blood and lymph system. IDO expression and activity are upregulated in numerous haematological malignancies [Table 3]. Hoshi et al. investigated the expression of IDO in Adult T-cell leukaemia/lymphoma (ATLL) cell and the chemotherapeutic effect on IDO-initiating L-Tryptophan catabolism in ATLL patients. Level of IDO mRNA expression and enzymatic activity of IDO in ATLL cells were noticeably enhanced in ATLL patients compared to healthy individuals. IDO was strongly expressed at tissue level as well. ${ }^{[34]}$ Lindström et al. determined activity and expression of IDO for chronic lymphocytic leukaemia (CLL) in 49 patients. Enhanced activity of IDO was observed in the CLL patients as compared to control, but in peripheral blood mononuclear cells mostly representing the malignant B cells, the gene expression of IDO1 and IDO2 was reduced. They found that IDO activity in CLL patients is associated with disease progression, even though it originates from cells other than malignant B cells. ${ }^{[35]}$

A comprehensive study conducted to investigate the IDO1 expression and function in 21 children with acute myeloid leukaemia (AML), [10 AML, 9 B-cell precursor (BCP)ALL, one infant acute leukaemia with MLL rearrangement and 1 T-cell ALL] and in one patient with $\mathrm{Ph}^{+}$chronic myeloid leukaemia. IDO activity was measured by reverse phase-HPLC. They found functional IDO1 expression in blast cells from of childhood AML subset, but not those from BCP-ALL or T-cell ALL. STAT3 inhibitors may effectively disrupt IDO1 expression by AML cells, thus in favour of anti-leukaemia immune responses tipping T helper type $1 / 2$ (Th1/Th2) equilibrium. ${ }^{[36]}$ Nakamura et al. explored the therapeutic potential of IDO inhibitor, D-1MT with cyclophosphamide (CY) using an IDOpositive B-cell lymphoma mouse model. D-1MT in

Table 3: Indoleamine 2,3-dioxygenase involvement in haematological malignancies

\begin{tabular}{|c|c|c|}
\hline Year & Investigator & Description \\
\hline 2009 & Hoshi et al. ${ }^{[34]}$ & $\begin{array}{l}\text { IDO is highly expressed in adult T-cell leukaemia/lymphoma. IDO-initiating L-tryptophan } \\
\text { catabolism changes with chemotherapy }\end{array}$ \\
\hline 2012 & Lindström et al. ${ }^{[35]}$ & $\begin{array}{l}\text { High IDO activity was observed in chronic lymphocytic leukaemia patients as compared to } \\
\text { control. Increased IDO activity may affect disease progression }\end{array}$ \\
\hline 2012 & Folgiero et al. ${ }^{[36]}$ & $\begin{array}{l}\text { IDO1 is expressed by a subset of childhood acute myeloid leukaemia and restrains IFN- } \gamma \text { pro- } \\
\text { duction by T-cells }\end{array}$ \\
\hline 2013 & Nakamura et al..$^{[37]}$ & $\begin{array}{l}\text { IDO inhibitor in combination with cyclophosphamide is an effective treatment for IDO-positive } \\
\text { lymphoma in a mouse model. IDO inhibition might offer a favourable treatment strategy for } \\
\text { lymphoma }\end{array}$ \\
\hline 2014 & Liu et al. ${ }^{[38]}$ & $\begin{array}{l}\text { IDO1 upregulation in non-Hodgkin lymphoma tissues could induce local immune tolerance by } \\
\text { infiltration of FoxP3+T-regs in the tumour microenvironment. This could be a novel mecha- } \\
\text { nism of immune escape }\end{array}$ \\
\hline 2014 & Choe et al. ${ }^{[39]}$ & $\begin{array}{l}\text { IDO is associated with the adverse clinical outcomes in (HL). IDO is an independent prognostic } \\
\text { factor in nodular sclerosis } \mathrm{HL}\end{array}$ \\
\hline 2014 & Folgiero et al. ${ }^{[40]}$ & $\begin{array}{l}\text { IDO1 expression by leukaemia blasts negatively affects the prognosis of childhood (AML). } \\
\text { IDO can be targeted, in adjunct to current chemotherapy strategies, to improve the clinical } \\
\text { outcome of children with AML }\end{array}$ \\
\hline 2016 & Mansour et al. ${ }^{[41]}$ & $\begin{array}{l}\text { High IDO expression in mesenchymal stem cells and increased levels of T-regs play pivotal role } \\
\text { in the pathogenesis of AML }\end{array}$ \\
\hline
\end{tabular}

IDO: Indoleamine 2,3-dioxygenase, $\mathrm{HL}$ : Hodgkin lymphoma, AML: Acute myeloid leukaemia, IFN- $\gamma$ : Interferon gamma 
combination with $\mathrm{CY}$ was recommended by them as an effective IDO-positive lymphoma treatment. These findings suggest that inhibition of IDO might offer a therapeutic approach for lymphoma. ${ }^{[37]}$

A recently published study demonstrated the role of IDO in non-Hodgkin lymphoma (NHL). They identified IDO1 upregulation in NHL tissues. They suggested that IDO has a critical role in immune escape mechanism in NHL. ${ }^{[38]}$ IDO is not only involved in the non-HL pathogenesis but also the HL. A study conducted by Choe et al. described IDO role in the HL microenvironment. They identified HL tissues with IDO positive cells. This study was the first of its kind in defining IDO as a principle immune-modulator and its involvement to adverse clinical outcomes of HL. ${ }^{[39]}$ A research published by Folgiero et al., in 2014, which was the continuation of his previous work published in 2012, suggested expression of IDO1 by leukaemia blasts adversely affects childhood AML prognosis. The 8-year event-free survival from a clinical standpoint was markedly worse in IDO-expressing children as compared to nonexpressing ones. Chemotherapeutic agents in combination with IDO inhibitors were proposed by them to improve the clinical outcomes in the children with AML. ${ }^{[40]}$ Another study on AML revealed that elevated level of T-regs has relationship with enhanced expression of IDO in mesenchymal stem cells which, in turn, responsible for immunosuppression in the tumour microenvironment. IDO has the main role in the pathogenesis of AML. ${ }^{[1]}$

\section{IDO in Prostate Cancer}

Prostate cancer is the most commonly diagnosed malignancy and a leading cause of mortality worldwide. ${ }^{[42]}$ Overexpression of IDO gene was observed in the prostate cancer patients [Table 4]. Feder-Mengus et al. evaluated the expression of IDO and few other genes in benign prostatic hyperplasia $(\mathrm{BPH})$ and prostate-specific antigen (PCA) tissues in 76 patients. IDO gene expression was quantitatively higher $(P=0.00001)$ and more repeatedly detectable $(P=0.00007)$ in PCA tissues in comparison with BPH. In serum analysis of these patients indicated an association between kynurenine/tryptophan ratio and IDO gene expression. ${ }^{[43]}$ The same kind of results identified by Provenzano et al. that IDO has potential to use as biomarker of malignant transformation in prostate cancers and warranted further research in PCA patients. ${ }^{[44]}$

Gray et al., in 2009, identified the upregulation of tumour IDO and transforming growth factor-beta in more advanced prostate cancer. They proposed that immunotherapy clinical trials in prostate cancer patients will be more fruitful if conducted in less advanced disease. ${ }^{[45]}$ Matos et al. pointed out the role of IDO in prostate cancer and suggested that IDO inhibition has clinical implication in prostate cancer. ${ }^{[46]}$

\section{IDO in Pancreatic Cancer}

IDO upregulation is associated with an increased number of T-regs in metastatic pancreatic ductal adenocarcinoma cells. $^{[47]}$ T-regs are key mediators of peripheral tolerance and have immunosuppressive activity. ${ }^{[48]}$ Low numbers of T-regs in pancreatic tumours were found to have a markedly better survival in comparison with high T-regs number in tumours. ${ }^{[49]}$ Kobayashi et al. investigated the localization and prevalence of $\mathrm{CD}^{+}$lymphocytes, IDO expression and $\mathrm{FOXP}^{+}$T-regs in intraductal papillary mucinous neoplasms (IPMNs) by immunohistochemistry in 39 cases. They observed IDO expression in the tumour

\section{Table 4: Indoleamine 2,3-dioxygenase involvement in prostate cancer}

\begin{tabular}{l|l|l}
\hline Year & Investigator & Description \\
\hline 2008 & Feder-Mengus et al. ${ }^{[43]}$ & High expression of IDO gene was explicitly detectable in (PCA) \\
\hline 2008 & Provenzano et al. ${ }^{[44]}$ & $\begin{array}{l}\text { IDO gene expression, recurrently detectable in PCA and associated with (AMACR) expres- } \\
\text { sion, seems to qualify as a marker of malignant transformation in prostate cancers }\end{array}$ \\
\hline 2009 & Gray et al. ${ }^{[45]}$ & $\begin{array}{l}\text { IDO, TGF- } \beta \text { upregulation and induction of T-regs in an immunosuppressive intratumoral } \\
\text { cytokine milieu identified in more advanced prostate cancer }\end{array}$ \\
\hline 2016 & Matos et al. ${ }^{[46]}$ & $\begin{array}{l}\text { IDO is produced constitutively by PC3 (human prostate cancer cell line) cells and its ex- } \\
\text { pression increase when the cells are exposed to IFN- } \gamma\end{array}$ \\
\hline
\end{tabular}

IDO: Indoleamine 2,3-dioxygenase, PCA: Prostate-specific antigen, AMACR: Alpha-methylacyl-CoA racemace, TGF- $\beta$ : Transforming growth factor beta 
Table 5: Indoleamine 2,3-dioxygenase involvement in pancreatic cancer

\begin{tabular}{l|l|l}
\hline Year & Investigator & Description \\
\hline 2008 & Witkiewicz et al. ${ }^{[4]]}$ & IDO inhibition in (PDA) patients can be useful to enhance immunotherapeutic strategies \\
\hline 2010 & Kobayashi et al. ${ }^{[50]}$ & $\begin{array}{l}\text { IDO expression in the tumor is one of the late-stage phenomena of multistage carcinogenesis } \\
\text { of (IPMNs) }\end{array}$ \\
\hline 2013 & Ikemoto et al. ${ }^{[51]}$ & $\begin{array}{l}\text { IDO-positive cells had a significantly higher recurrence than those with less IDO-positive cells. } \\
\text { Pathological aggressiveness of IPMNs is associated with IDO induced FOXP3 + T-regs }\end{array}$ \\
\hline 2014 & Peng et al. ${ }^{[53]}$ & $\begin{array}{l}\text { Elevation of (MMP-9) and IDO-induced by pancreatic cancer cells mediates (NK) cell dysfunc- } \\
\text { tion. MMP-9 and IDO facilitate pancreatic cancer cells to evade immunosurveillance }\end{array}$ \\
\hline
\end{tabular}

PDA: Pancreatic ductal adenocarcinoma, IDO: Indoleamine 2,3-dioxygenase, IPMNs: Intraductal papillary mucinous neoplasms, MMP-9: Matrix metallopeptidase 9, NK: Natural killer

was positively correlated with the prevalence of T-regs in IPMNs. ${ }^{[50]}$ Another study conducted by Ikemoto et al. to evaluate weather IDO and FOXP3 ${ }^{+}$T-regs interaction play a role in IPMN in pathological aggressiveness. They observed that the IDO-positive patient cells had a considerably higher recurrence than those with less IDO-positive cells. The aggressiveness of IPMNs is correlated with the involvement of FOXP $3^{+}$cells. IDO-positive cells in IPMNs can induce an increase in the FOXP3+ cells. ${ }^{[51]}$ Natural Killer (NK) cells are key components in the innate immune system and are capable of destroying the cancer cells directly. Dysfunction of NK cells has been investigated in pancreatic cancer ${ }^{[52]}$ Induction of IDO by pancreatic cancer cells promotes NK cell dysfunction. ${ }^{[53]}$ IDO helps pancreatic cancer cells to evade immunosurveillance ${ }^{[53]}$ [Table 5].

\section{IDO as an Immunotherapeutic Target}

IDO is involved in immune system regulation. IDO upregulation is associated with poor prognosis in various cancers $^{[20,30,34,35,39,41,43,51]}$ but few studies suggested that increased expression of IDO was linked with favourable prognosis. ${ }^{[19,46]}$ Further studies are required in view of the contradictory findings, to comprehend this complex role of IDO in various cancers. At present, in clinical stage, three IDO inhibitors are in commercial production as immunotherapeutic agents in breast cancer treatment. These include indoximod (NLG2101) developed by NewLink Genetics ${ }^{\text {TM }}$, ${ }^{[54]}$ INCB024360 developed by Incyte $^{\mathrm{TM}[55,56]}$ and NLG919 licensed to Genentech ${ }^{\mathrm{TM}}$. $^{[57]}$ Two therapies (NCT01042535 and NCT01792050) have been exclusively designed to treat HER2-positive breast cancer in combination with AD.p53 DC vaccine and docetaxel, respectively. ${ }^{[58]}$ Another immunotherapy is available for patients with metastatic pancreatic cancer
(NCT02077881). ${ }^{[59,60]}$ NCT01560923 is currently approved for the individuals with refractory metastatic prostate carcinoma. ${ }^{[61,62]}$ IDO-2 is a recently discovered IDO isoform. ${ }^{[63]}$ Future studies on the role of IDO-2 should be focused as well in cancer. Therapeutic implications of IDO have significant outcomes.

\section{Evidence Acquisition}

The search strategy used the following keywords: 'Immunotherapy' or 'IDO' or 'Breast cancer' or 'Colon cancer' or 'Haematological malignancies' or 'Prostate cancer' or 'Pancreatic cancer.' Three electronic databases (PubMed/MEDLINE, SCOPUS and GOOGLE SCHOLAR) were searched for articles published between 2008 and 2017. The most relevant articles were extracted from the literature.

\section{Conflict of Interest}

The authors declare that they have no conflict of interest.

\section{References}

1. Oiseth SJ, Aziz MS. Cancer immunotherapy: A brief review of the history, possibilities, and challenges ahead. J Cancer Metastasis Treat 2017;3:250-61.

2. Farkona S, Diamandis EP, Blasutig IM. Cancer immunotherapy: The beginning of the end of cancer? BMC Med 2016;14:73.

3. Challis GB, Stam HJ. The spontaneous regression of cancer. A review of cases from 1900 to 1987. Acta Oncol 1990;29:545-50.

4. Mansfield AS, Heikkila PS, Vaara AT, et al. Simultaneous foxp3 and IDO expression is associated with sentinel lymph node metastases in breast cancer. BMC Cancer 2009;9:231.

5. Muller AJ, DuHadaway JB, Donover PS, Sutanto-Ward E, Prendergast GC. Inhibition of indoleamine 2,3-dioxygenase, an immunoregulatory target of the cancer suppression 
gene bin1, potentiates cancer chemotherapy. Nat Med 2005;11:312-9.

6. Katz JB, Muller AJ, Prendergast GC. Indoleamine 2,3-dioxygenase in T-cell tolerance and tumoral immune escape. Immunol Rev 2008;222:206-21.

7. Munn DH, Sharma MD, Baban B, et al. GCN2 kinase in $\mathrm{T}$ cells mediates proliferative arrest and anergy induction in response to indoleamine 2,3-dioxygenase. Immunity 2005;22:633-42.

8. Ogata S, Takeuchi M, Fujita H, et al. Apoptosis induced by nicotinamide-related compounds and quinolinic acid in HL-60 cells. Biosci Biotechnol Biochem 2000;64:327-32.

9. Nakamura T, Shima T, Saeki A, et al. Expression of indoleamine 2,3-dioxygenase and the recruitment of foxp3-expressing regulatory $\mathrm{T}$ cells in the development and progression of uterine cervical cancer. Cancer Sci 2007;98:874-81.

10. Grohmann U, Fallarino F, Puccetti P. Tolerance, DCs and tryptophan: Much ado about IDO. Trends Immunol 2003;24:242-8.

11. Moffett JR, Espey MG, Namboodiri MA. Antibodies to quinolinic acid and the determination of its cellular distribution within the rat immune system. Cell Tissue Res 1994;278:461-9.

12. Yoshida R, Park SW, Yasui H, Takikawa O. Tryptophan degradation in transplanted tumor cells undergoing rejection. J Immunol 1988;141:2819-23.

13. Malina HZ, Martin XD. Indoleamine 2,3-dioxygenase: Antioxidant enzyme in the human eye. Graefes Arch Clin Exp Ophthalmol 1996;234:457-62.

14. Hwu P, Du MX, Lapointe R, Do M, Taylor MW, Young HA, et al. Indoleamine 2,3-dioxygenase production by human dendritic cells results in the inhibition of T cell proliferation. J Immunol 2000;164:3596-9.

15. Munn DH, Sharma MD, Lee JR, et al. Potential regulatory function of human dendritic cells expressing indoleamine 2,3-dioxygenase. Science 2002;297:1867-70.

16. Godin-Ethier J, Hanafi LA, Piccirillo CA, Lapointe R. Indoleamine 2,3-dioxygenase expression in human cancers: Clinical and immunologic perspectives. Clin Cancer Res 2011;17:6985-91.

17. Pardoll DM. The blockade of immune checkpoints in cancer immunotherapy. Nat Rev Cancer 2012;12:252-64.

18. Sadun RE, Sachsman SM, Chen X, et al. Immune signatures of murine and human cancers reveal unique mechanisms of tumor escape and new targets for cancer immunotherapy. Clin Cancer Res 2007;13:4016-25.

19. Jacquemier J, Bertucci F, Finetti $\mathrm{P}$, et al. High expression of indoleamine 2,3-dioxygenase in the tumour is associated with medullary features and favourable outcome in basallike breast carcinoma. Int J Cancer 2012;130:96-104.

20. Levina V, Su Y, Gorelik E. Immunological and nonimmunological effects of indoleamine 2,3-dioxygenase on breast tumor growth and spontaneous metastasis formation. Clin Dev Immunol 2012;2012:173029.
21. Soliman H, Rawal B, Fulp J, et al. Analysis of indoleamine 2-3 dioxygenase (IDO1) expression in breast cancer tissue by immunohistochemistry. Cancer Immunol Immunother 2013;62:829-37.

22. Zheng X, Koropatnick J, Chen D, et al. Silencing IDO in dendritic cells: A novel approach to enhance cancer immunotherapy in a murine breast cancer model. Int J Cancer 2013;132:967-77.

23. Yu J, Du W, Yan F, et al. Myeloid-derived suppressor cells suppress antitumor immune responses through IDO expression and correlate with lymph node metastasis in patients with breast cancer. J Immunol 2013;190:3783-97.

24. Isla Larrain MT, Rabassa ME, Lacunza E, Barbera A, Cretón A, Segal-Eiras A, et al. IDO is highly expressed in breast cancer and breast cancer-derived circulating microvesicles and associated to aggressive types of tumors by in silico analysis. Tumour Biol 2014;35:6511-9.

25. Salvadori ML, da Cunha Bianchi PK, Gebrim LH, et al. Effect of the association of 1-methyl-DL-tryptophan with paclitaxel on the expression of indoleamine 2,3-dioxygenase in cultured cancer cells from patients with breast cancer. Med Oncol 2015;32:248.

26. Chen Y, Xia R, Huang Y, et al. An immunostimulatory dual-functional nanocarrier that improves cancer immunochemotherapy. Nat Commun 2016;7:13443.

27. Kim S, Park S, Cho MS, et al. Strong correlation of indoleamine 2,3-dioxygenase 1 expression with basal-like phenotype and increased lymphocytic infiltration in triplenegative breast cancer. J Cancer 2017;8:124-30.

28. Noonepalle SK, Gu F, Lee EJ, et al. Promoter methylation modulates indoleamine 2,3-dioxygenase 1 induction by activated $\mathrm{T}$ cells in human breast cancers. Cancer Immunol Res 2017;5:330-44.

29. Engin A, Gonul II, Engin AB, et al. Relationship between indoleamine 2,3-dioxygenase activity and lymphatic invasion propensity of colorectal carcinoma. World J Gastroenterol 2016;22:3592-601.

30. Ferdinande L, Decaestecker C, Verset L, et al. Clinicopathological significance of indoleamine 2,3-dioxygenase 1 expression in colorectal cancer. Br J Cancer 2012;106:141-7.

31. Thaker AI, Rao MS, Bishnupuri KS, et al. IDO1 metabolites activate $\beta$-catenin signaling to promote cancer cell proliferation and colon tumorigenesis in mice. Gastroenterology 2013;145:416-250.

32. Eyraud D, Granger B, Zahr N, et al. Measurement of indoleamine 2, 3 dioxygenase activity in colorectal cancer: A Controlled group study. J Clin Cell Immunol 2015;6:374.

33. Chen IC, Lee KH, Hsu YH, et al. Expression pattern and clinicopathological relevance of the indoleamine 2,3-dioxygenase 1/Tryptophan 2,3-dioxygenase protein in colorectal cancer. Dis Markers 2016;2016:8169724.

34. Hoshi $\mathrm{M}$, Ito $\mathrm{H}$, Fujigaki $\mathrm{H}$, et al. Indoleamine 2,3-dioxygenase is highly expressed in human adult T-cell leukemia/lymphoma and chemotherapy changes tryptophan 
catabolism in serum and reduced activity. Leuk Res 2009;33:39-45.

35. Lindström V, Aittoniemi J, Jylhävä J, et al. Indoleamine 2,3-dioxygenase activity and expression in patients with chronic lymphocytic leukemia. Clin Lymphoma Myeloma Leuk 2012;12:363-5.

36. Folgiero V, Natale D, Pagliara D, et al. Indoleamine 2, 3-dioxygenase-1 (IDO1) Is expressed by a subset of childhood acute myeloid leukemias and restrains IFN- $\gamma$ production by T cells. Blood 2012;120:21.

37. Nakamura N, Hara T, Shibata Y, et al. Combination of indoleamine 2,3-dioxygenaseinhibitorand cytotoxic agents is a novel therapeutic option for non-hodgkin lymphoma. Blood 2013;122:21.

38. Liu XQ, Lu K, Feng LL, et al. Up-regulated expression of indoleamine 2,3-dioxygenase 1 in non-hodgkin lymphoma correlates with increased regulatory T-cell infiltration. Leuk Lymphoma 2014;55:405-14.

39. Choe JY, Yun JY, Jeon YK, et al. Indoleamine 2,3-dioxygenase (IDO) is frequently expressed in stromal cells of hodgkin lymphoma and is associated with adverse clinical features: A retrospective cohort study. BMC Cancer 2014;14:335.

40. Folgiero V, Goffredo BM, Filippini P, et al. Indoleamine 2,3-dioxygenase 1 (IDO1) activity in leukemia blasts correlates with poor outcome in childhood acute myeloid leukemia. Oncotarget 2014;5:2052-64.

41. Mansour I, Zayed RA, Said F, et al. Indoleamine 2,3-dioxygenase and regulatory $\mathrm{T}$ cells in acute myeloid leukemia. Hematology 2016;21:447-53.

42. Wong MC, Goggins WB, Wang HH, et al. Global incidence and mortality for prostate cancer: Analysis of temporal patterns and trends in 36 countries. Eur Urol 2016;70:862-74.

43. Feder-Mengus $\mathrm{C}$, Wyler $\mathrm{S}$, Hudolin $\mathrm{T}$, et al. High expression of indoleamine 2,3-dioxygenase gene in prostate cancer. Eur J Cancer 2008;44:2266-75.

44. Provenzano M, Feder-Mengus C, Wyler S, et al. Indoleamine 2,3- dioxygenase (IDO) expression and malignant transformation in prostate cancer. J Clin Oncol 2008;26:15.

45. Gray A, de la Luz Garcia-Hernandez M, van West M, et al. Prostate cancer immunotherapy yields superior long-term survival in TRAMP mice when administered at an early stage of carcinogenesis prior to the establishment of tumorassociated immunosuppression at later stages. Vaccine 2009;27 Suppl 6:G52-9.

46. Matos YS, Brito RB, Matheus LS, et al. The role of indoleamine 2,3-dioxygenase (IDO) on pc-3 prostate cancer cells viability. FASEB J 2016;30:698-718.

47. Witkiewicz A, Williams TK, Cozzitorto J, et al. Expression of indoleamine 2,3-dioxygenase in metastatic pancreatic ductal adenocarcinoma recruits regulatory $\mathrm{T}$ cells to avoid immune detection. J Am Coll Surg 2008;206:849-54.

48. Watanabe MA, Oda JM, Amarante MK, et al. Regulatory T cells and breast cancer: Implications for immunopathogenesis. Cancer Metastasis Rev 2010;29:569-79.
49. Ikemoto T, Yamaguchi T, Morine Y, et al. Clinical roles of increased populations of foxp $3+\mathrm{CD} 4+\mathrm{T}$ cells in peripheral blood from advanced pancreatic cancer patients. Pancreas 2006;33:386-90.

50. Kobayashi N, Kubota K, Kato S, et al. FOXP3+ regulatory $\mathrm{T}$ cells and tumoral indoleamine 2,3-dioxygenase expression predicts the carcinogenesis of intraductal papillary mucinous neoplasms of the pancreas. Pancreatology 2010;10:631-40.

51. Ikemoto T, Shimada M, Komatsu M, et al. Indoleamine 2,3-dioxygenase affects the aggressiveness of intraductal papillary mucinous neoplasms through foxp3+CD4+CD25+ T cells in peripheral blood. Pancreas 2013;42:130-4.

52. Duan X, Deng L, Chen X, et al. Clinical significance of the immunostimulatory MHC class I chain-related molecule A and NKG2D receptor on NK cells in pancreatic cancer. Med Oncol 2011;28:466-74.

53. Peng YP, Zhang JJ, Liang WB, et al. Elevation of MMP-9 and IDO induced by pancreatic cancer cells mediates natural killer cell dysfunction. BMC Cancer 2014;14:738.

54. Metz R, Rust S, Duhadaway JB, et al. IDO inhibits a tryptophan sufficiency signal that stimulates mTOR: A novel IDO effector pathway targeted by D-1-methyl-tryptophan. Oncoimmunology 2012;1:1460-8.

55. Liu X, Shin N, Koblish HK, et al. Selective inhibition of IDO1 effectively regulates mediators of antitumor immunity. Blood 2010;115:3520-30.

56. Koblish HK, Hansbury MJ, Bowman KJ, et al. Hydroxyamidine inhibitors of indoleamine-2,3-dioxygenase potently suppress systemic tryptophan catabolism and the growth of IDO-expressing tumors. Mol Cancer Ther 2010;9:489-98.

57. Mautino MR, Jaipuri FA, Waldo J, et al. NLG919, a novel indoleamine-2,3-dioxygenase (IDO)-pathway inhibitor drug candidate for cancer therapy. Am Assoc Cancer Res 2013;73:8.

58. Moon YW, Hajjar J, Hwu P, et al. Targeting the indoleamine 2,3-dioxygenase pathway in cancer. J Immunother Cancer 2015;3:51.

59. Vonderheide RH, Burg JM, Mick R, et al. Phase I study of the CD40 agonist antibody CP-870,893 combined with carboplatin and paclitaxel in patients with advanced solid tumors. Oncoimmunology 2013;2:e23033.

60. Hoffmann J, Vitale I, Buchmann B, et al. Improved cellular pharmacokinetics and pharmacodynamics underlie the wide anticancer activity of sagopilone. Cancer Res 2008; 68:5301-8.

61. Galluzzi L, Senovilla L, Vacchelli E, et al. Trial watch: Dendritic cell-based interventions for cancer therapy. Oncoimmunology 2012;1:1111-34.

62. Vacchelli E, Vitale I, Eggermont A, et al. Trial watch: Dendritic cell-based interventions for cancer therapy. Oncoimmunology 2013;2:e25771.

63. Ball HJ, Yuasa HJ, Austin CJ, et al. Indoleamine 2,3-dioxygenase-2; a new enzyme in the kynurenine pathway. Int J Biochem Cell Biol 2009;41:467-71. 